EESTI NSV TEADUSTE AKADEEMIA TOIMETISED, 31. KOIDË FOOSIKA * MATEMAATIKA. 1982, NR. 2

ИЗВЕСТИЯ АКАДЕМИИ НАУК ЭСТОНСКОЙ ССР. ТОМ 31 ФИЗИКА * МАТЕМАТИКА. 1982, № 2

Р. П. ТАМКИВИ

\title{
ИССЛЕДОВАНИЕ ГОМОПЕРЕНОСА ЭНЕРГИИ \\ С ПОМОЩЬЮ СПЕКТРАЛЬНО СЕЛЕКТИВНОЙ КИНЕТИКИ НИЗКОТЕМПЕРАТУРНОЙ ФЛУОРЕСЦЕНЦИИ ХЛОРОФИЛЛОВ
}

Общепризнано, что одним из важнейших звеньев в цепи первичных процессов фотосинтеза является безызлучательный перенос энергии (ПЭ) электронного возбуждения различных форм хлорофилла. Поэтому исследования ПЭ в хлорофилловых растворах весьма необходимы для более полного понимания соответствующих процессов in vivo. Наше обращение к данной, довольно интенсивно изучаемой тематике (см., напр., обзор [ $\left.\left.{ }^{1}\right]\right)$ связано со следующими обстоятельствами.

Қак известно, межмолекулярный ПЭ обычно исследуется тремя методами, основанными на спектроскопически наблюдаемых явлениях концентрационного 1) тушения, 2) изменения закंона затухания и 3) деполяризации люминесценции. Последнее из них до сих пор было практически единственным источником сведений о гомопереносе энергии между химически идентичными молекулами. Однако сопоставление результатов измерения деполяризации с соответствующей теорией встречает известные трудности (см. $\left[{ }^{2}\right]$ ). Несостоятельность первых двух методов в случае гомопереноса связана с невозможностью выделения такого подмножества из одинаковых молекул, которое состояло бы преимущественно либо из доноров, либо из акцепторов энергии. Цель настоящего сообщения - продемонстрировать возможности изучения гомопереноса между молекулами хлорофилла (Хл) с помощью спектрально селективной низкотемпературной флуорометрии - т. е. с помощью модификации второго метода.

Как было неоднократно показано, спектры идентичных молекул в низкотемпературных твердых матрицах подвергаются статистическому разбросу по частотам $0-0$-перехода (рис. 1). Возникающее по этой

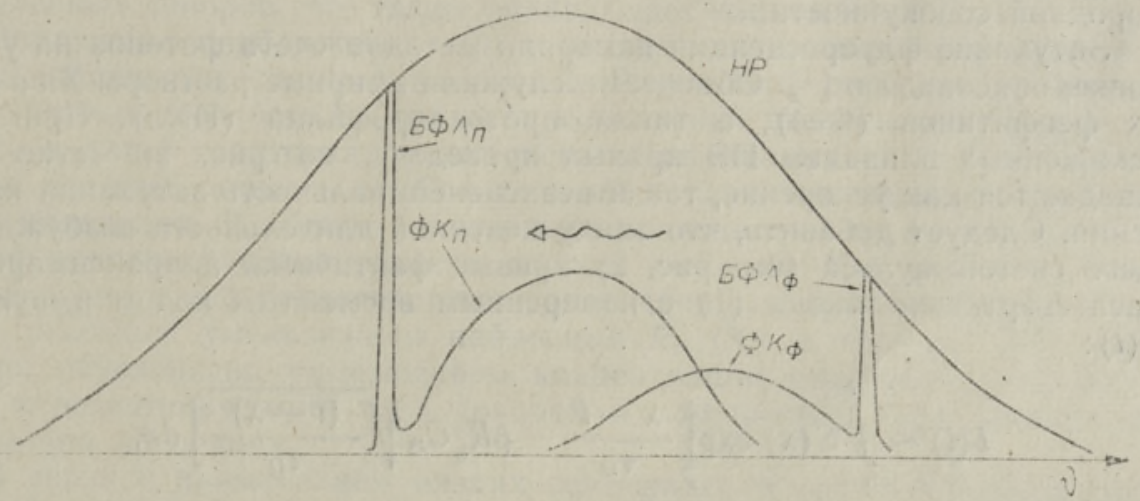

Рис. 1. Неоднородное распределение (НР) низкотемпературных однородных спектров молекул. Показаны бесфононные линии (БФЛ) и фононные крылья (ФК) спектров флуоресценции (Ф) и поглощения (П). Стрелкой указано направление ПЭ. 
причине неоднородное уширение спектров Хл достигает $\sim 300 \mathrm{~cm}^{-1}\left[{ }^{3}\right]$. Нами было проведено изучение времен затухания $\tau$ низкотемпературной флуоресценции Хл, монохроматически регистрируемой в пределах неоднородной полосы $\left[{ }^{4}\right]$. Обнаруженное концентрационное укорочение одноэкспоненциальных значений $\tau$ на коротковолновом краю полосы мы интерпретировали как проявление ферстеровского ПЭ вдоль неоднородного спектрального распределения молекул Хл. Как видно из рис. 1, молекулы, излучающие на коротковолновом краю полосы, участвуют в ПӘ в основном как доноры. Монохроматически регистрируемые кривые затухания свечения таких молекул должны содержать наиболее доступную информацию об этом процессе.

Закон затухания свечения доноров энергии в теории ФерстераГаланина [ $\left.{ }^{5}\right]$ можно записать так:

$$
I_{D}(t) \sim \exp \left(-\frac{t}{\tau_{D}}-\beta R_{0}^{3} C_{A} \sqrt{\frac{t}{\tau_{D}}}\right) .
$$

Здесь $\tau_{D}-$ время жизни возбужденного состояния доноров в отсутствие $П Э, C_{A}-$ концентрация акцепторов, $R_{0}-$ т. н. критический радиус ПЭ, служивший мерой эффективности ПЭ. $K_{0}$ пропорционален интегралу перекрывания спектра излучения доноров и спектра поглощения акцепторов. Коэффициент $\beta$ в случае полностью замороженных молекул равен $\approx 3,77 \cdot 10^{-3}\left(R_{0}\right.$ измеряется в $\AA$, а $C_{A}-$ в моль/ $\left.\Omega\right)\left[{ }^{2}\right]$.

Нетрудно видеть, что основные условия применимости теории Ферстера-Галанина в исследуемой нами ситуации выполнены. Однако непосредственному использованию формулы (1) препятствует неоднородный состав акцепторов. Как показано в [ $\left.{ }^{6}\right]$, тогда во втором члене экспоненты надо суммировать по всем сортам акцепторов $\left(R_{0}\right.$ и $C_{A}$ зависят от природы акцептора), что в нашем случае сводится к интегралу по неоднородному распределению. Даже для весьма простых моделей это ведет к довольно сложным и трудноинтерпретируемым выражениям. * Отметим, что поскольку последние содержат параметры как однородных, так и неоднородного спектров (см. рис. 1), такой подход может давать информацию о самом неоднородном распределении.

В данной работе мы аппроксимировали измеренные на коротковолновом спаде полосы флуоресценции кривые затухания формулой для однородного состава акцепторов, отдавая себе отчет в том, что определяемый таким образом $R_{0}$ соответствует взвешенной сумме интегралов перекрывания по неоднородному распределению акцепторов. Другими словами, наш $R_{0}$ представляет собой меру эффективности ПЭ от спектрально выделенных молекул ко всем возможным акцепторам из неоднородной совокупности.

Затухание флуоресценции измеряли методом счета фотонов на установке, описанной в [ $\left.{ }^{4}\right]$. Объектами служили эфирные растворы Хл $a$ и $b$, их феофитинов (Фео), а также протохлорофилла (ПХл). Примеры искаженных влиянием ПЭ кривых приведены на рис. 2. Четко наблюдаются как ускорение, так и неэкспоненциальность затухания излучения. Следует добавить, что ввиду конечной длительности возбуждающего светоимпульса (см. рис. 2) кривые фактически аппроксимировались свертками закона (1) с измеренным временным ходом импульса $S(t)$ :

$$
I(t) \sim \int_{0}^{t} S(x) \exp \left[\frac{x-t}{\tau_{D}}-\beta R_{0}^{3} C_{A} \sqrt{\frac{(t-x)}{\tau_{D}}}\right] d x
$$

\footnotetext{
* Аналоги (1) в случае спектральной неоднородности выведены «из первичных принципов» для предела, когда неоднородная ширина $\ll k T$ (см. напр., [7] и ссылки там), т. е. для случая, обратного нашему.
} 
Рис. 2. Кривые затухания флуоресценции Хл $a(1,2)$, зарегистрированной при 660 нм. и ПХл $(3,4)$ при 618 н.м. Концентрации: $5 \cdot 10^{-6}(1,3), 2 \cdot 10^{-3}$ (2) и $1,2 \cdot 10^{-3}$ моль/л (4). Показана форма возбуждения (5). Сплошные линии - наилучшие аппроксимации с формулой (2).

причем за $\tau_{D}$ были взяты $\tau$, полученные на той же длине волны флуоресценции разбавленных растворов. **

Вместо $C_{A}$ мы везде пользовались полной концентрацией раствора $C$. Это оправдано, с одной стороны, выбором точки регистрации излучения (доля еще более «коротковолновых» молекул мала). С другой стороны, для большинства возможных акцепторов ПЭ определяется перекрыванием фононных крыльев (см. рнс. 1). Относительно широкие $\left(\sim 100 \mathrm{~cm}^{-1}\right)$ фононные крылья спектров Хл были экспериментально наблюдены методом выжигания провала $\left[{ }^{9}\right]$. Проведенный нами модельный расчет (где все изображенные на рис. 1 контуры были взяты гауссовыми) показал, что в случае характерных для Хл величин параметров однородных и неоднородного спектров существенный вклад в сумму интегралов перекрывания вносят практически все молекулы, 0 -0-переход которых лежит в длинноволновой стороне от спектрально выделенных доноров. Это также оправдывает наш выбор $C_{A}$.

Наилучшие приближения (2) к экспериментальным кривым показаны сплошными линиями на рис. 2. Результаты определения вышеизложенным способом $R_{0}$ для ряда соединений сведены в таблицу.

Если наша трактовка причин искажения кривых затухания верна, $R_{0}$ не должны зависеть от $C$, что и наблюдается в экспериментах на всех молекулах. В общем регистрация проводилась по возможности в самой коротковолновой области полосы свечения. Как видно из таблицы, сдвиг точки регистрации к более длинным волнам сопровождается заметным уменьшением найденных $R_{0}$ (Хл $a$, Фео $a$ ). Это, очевидно, обусловлено уменьшением концентрации акцепторов, подходяших к регистрируемому типу. доноров, и нарушением чисто донорного характера последних.

В связи с применением низких температур и новой методики следует с осторожностью относиться к сравнению численных значений

** Как показано в [8], некоторая зависимость $\tau$ даже в очень разбавленных растворах Хл от длины волны низкотемпературной флуоресценции сохраняется. 


\begin{tabular}{|c|c|c|c|c|}
\hline Молекула & $C$, моль $/ \Omega$ & $\lambda_{\text {per }}, \mu M$ & $T, K$ & $R_{0}, \AA$ \\
\hline \multirow[t]{2}{*}{ Хл $a$} & $2 \cdot 10^{-3}$ & $660 / 664$ & $\begin{array}{r}5 \\
20 \\
50\end{array}$ & $\begin{array}{l}64 / 61 \\
65 / 63 \\
66 / 63\end{array}$ \\
\hline & $5 \cdot 10^{-3}$ & 660 & $\begin{array}{r}5 \\
20 \\
50\end{array}$ & $\begin{array}{l}65 \\
65 \\
67\end{array}$ \\
\hline \multirow[t]{2}{*}{ ПХл } & $1,2 \cdot 10^{-3}$ & 618 & $\begin{array}{r}5 \\
50\end{array}$ & $\begin{array}{l}46 \\
47\end{array}$ \\
\hline & $2,5 \cdot 10^{-3}$ & 618 & 5 & 46 \\
\hline Хл $b$ & $\begin{array}{l}1,2 \cdot 10^{-3} \\
2,3 \cdot 10^{-3}\end{array}$ & 643 & 4,2 & $\begin{array}{l}51 \\
50\end{array}$ \\
\hline Феo $a$ & $\begin{array}{l}1 \cdot 10^{-3} \\
3 \cdot 10^{-3}\end{array}$ & $661 / 664$ & 4,2 & $\begin{array}{l}68 / 63 \\
67 / 64\end{array}$ \\
\hline$\Phi$ eo $b$ & $1,7 \cdot 10^{-3}$ & 647 & 4,2 & 50 \\
\hline
\end{tabular}

наших $R_{0}$ c ранее опубликованными. K тому же для последних характерен значительный разброс, причинами которого являются, по-видимому, неодинаковые экспериментальные условия (методика, растворители, геометрия и т. п.), а возможно, и различные теоретические подходы к явлению деполяризации излучения $\left[{ }^{10}\right]$, служившему основным источником данных о гомопереносе энергии. В обзоре [ $\left.{ }^{1}\right]$ проанализированы сведения разных авторов о $R_{0}$ для Хл $a$ (в интервале значений от 42 до $92 \AA$ ) и предложено значение $R_{0} \approx 70 \AA$ в качестве наиболее для него правдоподобного. Следует отметить близость нашего $R_{0}$ к этому значению. Зависимость $R_{0}$ от соединения также согласуется с ранее известными данными. Так, например, в [ $\left.{ }^{11}\right]$ при $300 \mathrm{~K}$ получены следующие значения $R_{n}:$ Хл $a-50 \AA$, Хл $b-43 \AA$, Фео $a-52 \AA$ (ср. с таблицей). Найденный нами большой $R_{0}$ вновь подтверждает высокую эффективность ПЭ между молекулами Хл $a$ - главного фотосинтетического пигмента.

Была снята и температурная зависимость $R_{0}$ для Хл $a$ и ПХл. Как видно из таблицы, повышение температуры ведет к некоторому его росту. Такой, находящийся на пределе ошибок измерений рост может быть связан с увеличением эффективности ПЭ за счет включения актов ПЭ с участием. матричных фононов. Альтернативным объяснением может служить температурный сдвиг неоднородной полосы, влияющий на $R_{0}$ аналогично сдвигу длины волны регистрации излучения. Учет последней возможности при постановке эксперимента, в принципе, позволяет исследовать и участие колебаний в процессах ПЭ.

Итак, можно заключить, что развитый в данной работе подход открывает возможность как наблюдать, так и количественно исследовать ПЭ в системах с большим неоднородным уширением, в том числе и в замороженных растворах хлорофиллоподобных молекул. Уместно упомянуть еще о применении его к детектированию ПЭ для Хл in vivo $\left[{ }^{12}\right]$, хотя количественная трактовка в этом случае остается пока предметом будущих исследований.

Автор благодарен Р. А. Авармаа за постоянный йнтерес к данной работе и ценные замечания, а также С. А. Куузик и В. И. Нымм за очистку и приготовление образцов, 


\section{Л И ТЕРА Т Р А}

1. Knox, R. S., In: Primary Molecular Events in Photobiology, Elsevier, AmsterdamLondon-New York, 1973, p. 45-77.

2. Агранович В. М., Гал анин М. Д., Перенос энергии электронного возбуждения в конденсированных средах, М., «Наука», 1978.

3. Avarmaa, R., Rebane, K., Stud. Biophys., 48, № 3, 209-218 (1975).

4. Т а м к и в и Р. П., А в а р м а а Р. А., Изв. АН СССР, сер. физ., 42, № 3, 568-572 (1978).

5. Förster, Т., Ann. Phys., 2, № 1-2, 55-75 (1948); Галаннн М. Д., Ж. эксперим. и теор. физ., 28, вып. 4, 485-495 (1955).

6. B l umen, A., M a nz, J., J. Chem. Phys., 71, № 11, 4694-4702 (1979).

7. Klafter, J., Silbey, R., J. Chem. Phys., 72, № 2, 843-848 (1980).

8. Avarmaa, R., Soovik, T., Tamkivi, R., Tõnissoo, V., Stud. Biophys., 65, № 3, 213-218 (1977).

9. Avarmaa, R., Mauring, K., Suisalu, A., Chem. Phys. Lett., 77, № 1, 88-92 (1981).

10. K n o x, R. S., Physica, 39, № 2-3, 361-368 (1968).

11. Gurinovitch, G. P., Losev, A. P., Z enkevitch, E. I., Spectrosc. Lett., 11, № 7, 493-511 (1978).

12. Avarmaa, R. A., Kochubey, S. M., Tamkivi, R. P., FEBS Lett,, 102, № 1, 139-142 (1979).

Ннститут физики

Акъадемии наук Эстонской ССР

R. P. TAMKIVI

\section{ENERGIA HOMOOLEKANDE UURIMINE \\ KLOROFULLIDE MADALATEMPERATUURILISE FLUORESTSENTSI SPEKTRAALSELT SELEKTIIVSE KINEETIKA ABIL}

Elektronergastuse energia kiirguseta homoülekanne klorofüllisarnaste molekulide vahel on jälgitav madalatemperatuurilise fluorestsentsi kineetika monokromaatilise registreerimise tee!. Registreerimislainepikkuse sobiv valik mittehomogeenselt laienenud kiirgusriba piires võimaldab otsest võrdlust Förster-Galanini teooriaga. On määratud rea ühendite kriitilised ülekanderaadiused ning hinnatud kõnealuse lähenemisviisi iseärasusi ja perspektiive.

\section{R. P. TAMKIVI}

\section{INVESTIGATION OF THE ENERGY HOMOTRANSFER VIA THE SPECTRALLY SELECTIVE KINETICS OF LOW-TEMPERATURE FLUORESCENCE OF CHLOROPHYLLS}

The homotransfer of electronic excitation energy between chlorophyll-like molecules is observable due to monochromatic registration of the kinetics of the low-temperature fluorescence. A suitable choice of the registration wavelength within the limits of inhomogeneously broadened emission band enables to realize a direct comparison with Förster-Galanin theory. The critical transfer radii for a number of compounds are determined. Features and perspectives of the approaçh are dișcussed. 